\title{
Isolation and functional characterization of MxCS1: a gene encoding a citrate synthase in Malus xiaojinensis
}

\author{
D.-G. HAN ${ }^{1,2}$, Y. WANG ${ }^{1}$, L. ZHANG ${ }^{1}$, L. MA ${ }^{1}$, X.-Z. ZHANG ${ }^{1}$, X.-F. XU ${ }^{1}$ and Z.-H. HAN ${ }^{1 *}$ \\ Institute for Horticultural Plants, China Agricultural University and Key Laboratory of Beijing Municipality \\ of Stress Physiology and Molecular Biology for Fruit Tree, Beijing 100193, P.R. China ${ }^{1}$ \\ College of Horticulture, Northeast Agricultural University, Haerbin, 150030, P.R. China ${ }^{2}$
}

\begin{abstract}
Iron is one of the essential micronutrients required by all living organisms. In this study, we isolated a gene encoding putative citrate synthase (CS) from Malus xiaojinensis, designated as MxCS1. The MxCS1 gene encodes a protein of 473 amino acid residues with a predicted molecular mass of $52.5 \mathrm{kDa}$ and a theoretical isoelectric point of 8.67. The expression of MxCS1 was enriched in the leaf rather than in phloem and root, however, its expression was hardly detected in the xylem. The gene expression was strongly induced by Fe stress treatment in the M. xiaojinensis seedlings. Over-expression of MxCS1 improved Fe deficiency tolerance in transgenic Arabidopsis. We argued that MxCS1 is a new member of the CS genes, and it may function as a regulator in response to iron stress in plants.
\end{abstract}

Additional key words: gene expression, iron deficiency, transgenic Arabidopsis.

\section{Introduction}

Iron is an essential element for all plants. Iron has poor solubility in most soil (Guerinot and Yi 1994), particularly in calcareous soil where the concentration of free $\mathrm{Fe}$ is far below concentration optimal for plant growth (Han et al. 1994a,b). Fe-deficiency induces chlorosis, which is a worldwide problem for crop production (Chouliaras et al. 2004). Iron deficiency, especially in North China, can largely limit the growth, yield and quality of apples. To avoid Fe deficiency, plants have developed adaptable mechanisms for the efficient absorption and use of $\mathrm{Fe}$ (Benderliev et al. 2003), e.g., plants produce more ferric reductase-oxidase (FRO) under Fe-deficiency, to reduce $\mathrm{Fe}^{\mathrm{III}}$ to $\mathrm{Fe}^{\mathrm{II}}$ (Li et al. 2006, Zhang et al. 2009). Nicotianamine (NA) can chelate $\mathrm{Fe}^{\text {II }}$ for the Fe transport through phloem (Stephan and Scholz 1993, Stephan et al. 1994). Additionally, citric acid can chelate $\mathrm{Fe}^{\mathrm{III}}$ and transport it through xylem (Cataldo et al. 1988) where the $\mathrm{pH}$ is about 5.5 - 6.0 (Hell and Stephan 2003). The Arabidopsis mutant, frd3, has provided molecular evidence of the role of citric acid in longdistance iron transport (Rogers and Guerinot 2002, Green and Rogers 2004, Durrett et al. 2007). However, the role of citrate synthase (CS) has not been determined yet.

To identify Fe-efficient genotypes, we have collected more than 40 samples from various species and ecotypes of genus Malus and indicated that M. xiaojinensis is a Fe-efficient apple genotype (Han et al. 1998). However, the role of CS in $M$. xiaojinensis, has not yet been confirmed. The $C S$ gene plays a key role in synthesizing $\mathrm{CS}$, but the relation between the $C S$ and iron transport remains unclear. In this study, we isolated a $C S$ gene from M. xiaojinensis, designated as MxCS1. We detected the expression of MxCS1 in different organs, and found relationship between the expression of MxCS1 and applied Fe concentration. The most importantly, we found that over-expression of the MxCS1 can improve tolerance to Fe deficiency in transgenic Arabidopsis.

Received 13 April 2010, accepted 21 September 2010.

Abbreviations: BA - 6-benzylaminopurine; CaMV - the cauliflower mosaic virus; CS - citrate synthase; IBA - indolebutyric acid; MS - Murashige and Skoog; ORF - open reading frame; RACE - rapid amplification of cDNA ends; RT-PCR - reverse transcriptasepolymerase chain reaction; WT - wild type.

Acknowledgements: This project was supported by the genetically modified organisms breeding major projects (2009ZX08009122B); New stars on Science and Technology of Beijing (2008B74); The National Natural Science Foundational of China (30800741) and Chinese universities scientific funds (2009-2-06).

* Corresponding author; fax: (+86) 01062734391 , e-mail: rschan@cau.edu.cn 


\section{Materials and methods}

Malus xiaojinensis (a wild apple rootstock, Han et al. 1994a) seedlings were rapidly propagated on Murashige and Skoog (MS) medium with $0.5 \mathrm{mg} \mathrm{dm}^{-3}$ 6-benzylaminopurine (BA) and $0.5 \mathrm{mg} \mathrm{dm}^{-3}$ indolebutyric acid (IBA) for one month, and then returned to $\mathrm{MS}+$ $1.0 \mathrm{mg} \mathrm{dm}^{-3}$ IBA for another and a half months for rooting. Finally the seedlings were grown in Hoagland nutrient solution for one month. Plants were exposed to different Fe concentration in Hoagland nutrient solutions $(4,40$ and $160 \mu \mathrm{M})$ when the plants have $8-9$ mature leaves (fully expanded).

Nested PCR was performed to obtain a partial sequence of MxCS1 by using the first strand cDNA of M. xiaojinensis as a template. Two degenerate primers corresponding to the amino acid sequences ELIPEQQE [5'-GAAT(A)TGATTCCAGAACAACAGGAA(C/G)C-3'] and C-terminal WPNVDAHS [5'-CACTGTGA(G)GCA TCT(A)ACATTG(T)GGCCA-3'] were used for the first PCR, and then the WPNVDAHS primer and another primer corresponding to the WETSLLDP sequence [5'-TGGGAAACCTC(A/G)CTTA(G)CTTGACCCA(G/T) GA-3'] were used for the second PCR.

For 3' rapid amplification of cDNA ends (RACE), two gene-specific primers were designed: GSP1, 5'-AGAATG CCAGAAAGTATTACCTGCT-3', and GSP2, 5'-TTG CACAAGTGCCAGTAGTAGCTGC-3'. For the 5'RACE, the primers GSP3, 5'-GCAGGTAATACTTTCTGG CATTCTG-3', and GSP4 5'-CATAAGCTGGAA CTACAGCACGAGT-3' were used. The RACE reactions were performed with RACE cDNA amplification kit (Invitrogen, Beijing, China) according to the manufacturer's instruction. A single, full-length, cDNA sequence was obtained by combining the 5'-RACE fragment, cDNA fragment and 3'-RACE fragment together. A pair of primers (F1, 5'-TTCACA TTTAAAACGTAATTGAGTT-3' and F2 5'-AGAAGT GCTTGGACTTTATTTTAAC-3') were designed to amplify the putative $5^{\prime}$ and $3^{\prime}$ untranslated region (UTR) of the full-length cDNA sequence. The resulting DNA fragments and RACE products were gel purified and cloned into the pMD18-T vector (Takara Biotechnology, Dalian, Japan) and sequenced (Invitrogen).

Total RNA was extracted separately from roots and leaves using the CTAB method (Zhang et al. 2005). First strand cDNA was synthesised with $1 \mu \mathrm{g}$ total RNA and $0.001 \mathrm{~cm}^{3}$ superscript II enzyme (Invitrogen) according to the protocol from manufacturer. As a control, the actin rRNA gene was amplified from $M$. xiaojinensis tissues using the following primers: ApAts1, 5'-CTACAA AGTCATCGTCCAGACAT-3' and ApAtR1, 5'-TGG

\section{Results}

By designing primers to the homologous regions of $\mathrm{CS}$, partial cDNA of MxCS1 gene was isolated from
GATGACATGGAGAAGATT-3'. The primers used for detecting MxCS1 gene expression were as follows: forward, 5'- GTATTCTTCAGGAGCGTCACC -3' and reverse, 5'-GCTCGGTCCCATATCAACTG-3'. All excised samples were frozen in liquid nitrogen and immediately stored at $-80{ }^{\circ} \mathrm{C}$ for RNA extraction. Samples including root, xylem, phloem and leaves (partly expanded new leaves) of normal Fe treatment $(40 \mu \mathrm{M})$; roots and leaves taken from treated plants under low $(4 \mu \mathrm{M})$ and high $(160 \mu \mathrm{M})$ Fe treatments for $0,12,24$ and $48 \mathrm{~h}$ respectively. The PCR was performed as follows: pre-denaturation at $94{ }^{\circ} \mathrm{C}$ for $3 \mathrm{~min}$, followed by 30 cycles of $40 \mathrm{~s}$ at $94^{\circ} \mathrm{C}, 40 \mathrm{~s}$ at $50^{\circ} \mathrm{C}, 90 \mathrm{~s}$ at $72{ }^{\circ} \mathrm{C}$ for MxCS1 (25 cycles for actin) and a final extension of $7 \mathrm{~min}$ at $72{ }^{\circ} \mathrm{C}$. The amplified products were detected using a $1.1 \%$ agarose gel electrophoresis. All RT-PCR experiments were repeated at least three times.

To construct an expression vector for transformation of Arabidopsis, restriction enzyme cut sites of $X b a \mathrm{I}$ and SacI were added into MxCS1 cDNA at both 5' and 3' end by PCR. To construct the pBI121-MxCS1 vector, the products of PCR and pBI121 were digested by $X b a \mathrm{I}$ and $S a c I$, and ligated together through the replacing of GUS gene. The MxCS1 gene driven under the cauliflower mosaic virus (CaMV) 35S promoter was introduced into Arabidopsis thaliana ecotype Columbia (Col-0) plants by Agrobacterium-mediated GV3101 transformation (An et al. 1988) using the vacuum infiltration method. Transformants were selected on MS medium containing $50 \mathrm{mg} \mathrm{dm}^{-3}$ kanamycin.

The $T_{3}$ generation plants were used in the subsequent experiments. For the growth assay, $T_{3}$ transgenic plants of lines, control plant only transformed only PBI121 vector line and wild-type seeds were placed on MS agar plates for germination. After $4 \mathrm{~d}, 30$ germinated seedlings from each line were carefully transferred to new MS agar plates supplemented with 4 (low), 100 (normal) or $400 \mu \mathrm{M}$ (high) $\mathrm{Fe}$ concentration. After $10 \mathrm{~d}$, the fresh mass of seedlings was measured. Three replicates were conducted and the standard errors were calculated.

Chlorophyll content was measured according to the method of Aono et al. (1993). Fe concentrations in leaves were measured according to Kojima and Iida (1986) and Takahashi et al. (2003). The CS activities in leaves were measured according to Bryan et al. (2001). Assays for the content of citric acid were performed by $o$-phthaldialdehyde (OPA) derivatisation, reverse-phase high performance liquid chromatography (HPLC) separation and fluorescence detection of the OPA derivatives. In all experiments pure citric acid was used (Sigma, St. Louis, USA) as an external standard (Zhang et al. 2009).

M. xiaojinensis using PCR. The full-length cDNA was obtained by employing rapid amplification of 3'-cDNA 
91 ATTCTTCAGGAGCGTCACCGCGCTTTCCAAGCTCCGTTCTCGTCTCGGGCAACTGTCGAGTCTCAGGGATTCCGTCAGATGGATTCAAAC

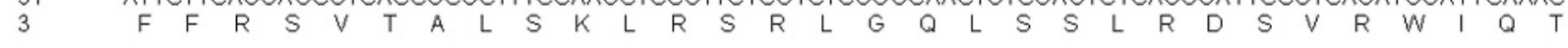

181 GCAGACCTCCACAGATCTCGACCTTCGTTCTCAGTTGGCGGAATTGATTCCAGAACAACAGGAACGTCTGAAAAAACTGAAGGCAGATTA

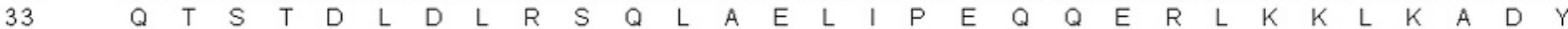

271 TGGAAAAGTTCAACTGGGCAATATCACGGTTGATATGGTGATTGGTGGAATGAGAGGAATGACAGGGTTGCTGTGGGAAACCTCCTTACT $63 \quad G \quad K \quad \vee \quad Q \quad L \quad G \quad N \quad I \quad T \quad V \quad D \quad M \quad V \quad I \quad G \quad G \quad M \quad R \quad G \quad M \quad T \quad G \quad L \quad L \quad W \quad E \quad T \quad S \quad L \quad L$

361 TGATCCAGATGAGGGAATTCGCTTCAGGGGTTTGTCAATTCCAGAATGCCAGAAAGTATTACCTGCTGCAAAGCCAGGTGGAGAACCTT

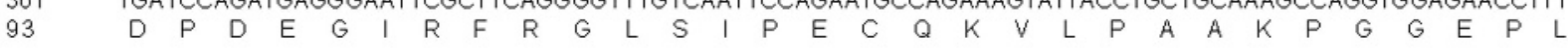

451 GCCTGAGGGTCTTCTGTGGCTGCTTTTGACAGGAAAGGTACCTAGCAAAGAGCAAGTAGATGCATTATCCAAGGAATTGAGGACTCGTGC

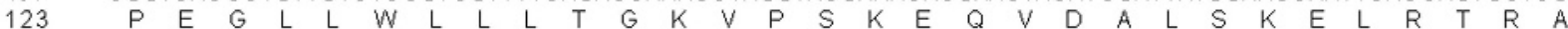

541 TGTAGTTCCAGCTTATGTGTATAAGGCCATTGATGCTCTGCCTATAACAGCACATCCAATGACCCAGTTCACCACTGGTGTCATGGCCCT

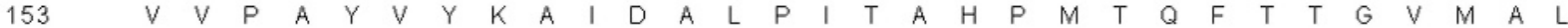

631 CCAGGTAGACAGTGAATTCCAGAAGGCATATGAAAAGGGGATACATAAATCAAAGTACTGGGAGCCAACTTTTGAGGATTCACTTAGCTT

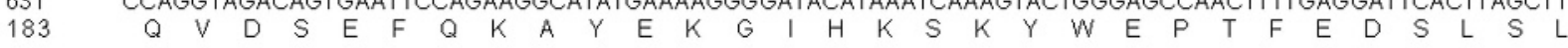

721 GATTGCACAAGTGCCAGTAGTAGCTGCCTATATTTATCGAAGAATTTTCAAGGATGGAAAAGTAAGACCTGTTAATGATTCTCTGGATTA

TGGTGCAAATTTTTCACACATGCTGGGTTTTGACGATCCCATAGTGCATGAGCTCATGAGGCTTTATGTCACCATCCATAGTGATCATGA

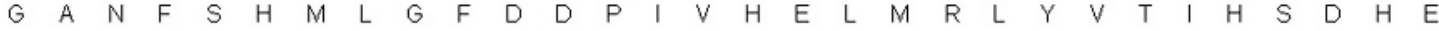
AGGTGGGAATGTCAGTGCTCACAOCGGCCACTTAGTTGCTAGTGCACTTTCAGATCCGTATCTTTCATTTGCAGCTGCTTTAAACGGCTT

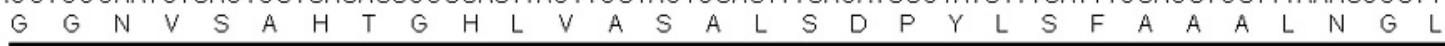
GGCTGGCCCACTCCATGGTTTGGCTAATCAGGAAGTATTGCTTTGGATAAAGTCTGTGGTAGATGAAGTTGGAGAGAATGTAACTACAAA

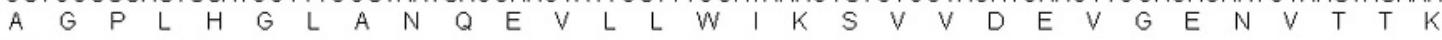
GCAGTTGAAAGATTATGTCTGGAAAACATTAAAAAGTGGGAAGGTTGTTCCTGGTTTTGGACACGGAGTCTTGCGTAAAACAGACCCACG

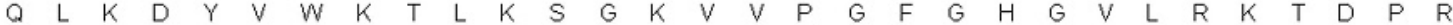
ATACACATGTCAAAGGGAGTTTGCATTGAAGCACATGCCTGATGATCCGCTGTTTCAGCTGGTCTCGAAGCTTTATGAAGTTGTGCCTCC

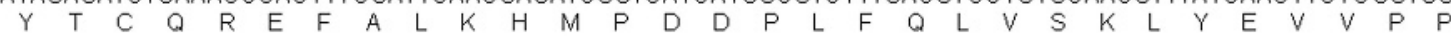
CGTACTTACTGAACTAGGCAAGGTTAAAAACCCATGGCCCAATGTAGATGCACACAGTGGAGTTCTGTTGAACCATTTGGTTTGACTGA

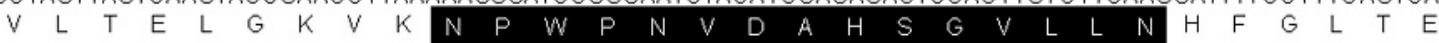

GGCAAGGTATTTTACTGTTCTTTTCGGTGTATCGAGGAGTATAGGGATTGGTTCCCAGTTGATATGGGACCGAGCTCTTGGTTTGCCTCT

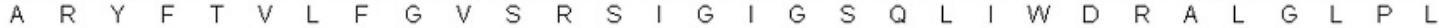
TGAAAGGCCAAAAAGTGTTACGATGGAATCGCTTGAGAATTTCTGCAAGAAAGCAGCTTCGTCTTGAGAAATTGAGTGCTGTATCACTCT E R P K S V T M E S L E N F C K K A A S

GAGTAATAATTGCTCCCGAATGATGGCTTTGTGAAAGATGCAACGGTTGTGTAAAAATTTCAGTTTCTGTTGGTTTTAGTTGAGAAATTG TTACAGGGAAACCTCAACACTCACACATCACACTTGAGCATTTTCAGGTACTTTAAAGAAATTTTGAAGTGAGATAGGCGTTGGGGCTTT TGCTGCTCGGTTAATAAACGGATTGGAGCTATTTAGATTAAAACGTGTACTTTAAAGATTTCCACAAGTAGTAAGCGTTTTACGCGTATT CTGCTATGAGGGCGGCACATTTATTATTGAGAACTTTAATGAGAAGTGCTTGGACTTTATTTTAACAAAAAAAAAAAAAAAA

Fig. 1. Nucleotide and deduced amino acid sequences of MxCS1 (GenBank accession No. HM459855). The citrate synthase domain is underlined, The PWPN-motif is highlighted with white letters in the black background.

end (3'-RACE) and 5'-cDNA end (5'-RACE). Sequence analysis showed that the MxCS1 cDNA was $1882 \mathrm{bp}$, including a complete open reading frame of $1422 \mathrm{bp}$ flanked by a 85 bp 5'-UTR and a 375 bp 3'-UTR (Fig. 1; Genbank accession No. HM459855). The predicted protein of MxCS1 comprises 473 amino acids with a predicted molecular mass of $52.5 \mathrm{kDa}$, and a theoretical isoelectric point of 8.67 .

To investigate the evolutionary relationship among plant CS proteins, 8 genes of CS proteins from different species were analysed by DNAman analyse software (Fig. 2). The deduced amino acid sequence of MxCS1 included one conserved $\mathrm{CS}$ domain in the C-terminal region (Fig. 2A) and a PWPN-box in the N-terminus (Fig. 2B) contained the plant-specific WPNVDAHS sequence which serves as a DNA-binding motif of CS (Christelle et al. 2002, Takita et al. 1999). The PWPNbox in the $\mathrm{N}$-terminus functions as a transcriptional repression domain.

Comparing the amino acid sequences of MxCS1 with the CS proteins from other species, we found that MxCS1 had a high identity to the CS protein family. Additionally, a phylogenetic tree (Neighbour-Joining) was constructed with the full-length amino acid residues (Fig. 2C). The results showed that MxCS1 from M. xiaojinensis and CS1 from peach (Christelle et al. 2002; AF367444.1) clustered together, followed by clustering of grapefruit (U19481.1) and orange (GQ372880.1). However, Arabidopsis (NM_129998.3), carrot (Takita et al. 1999; AB017159.1), rice (AF302906.1) and tobacco (X84226) 
were grouped into another cluster.

The expression profile of the MxCS1 in various M. xiaojinensis tissues was investigated using the semiquantitative RT-PCR. Expression of $M x C S 1$ was enriched in leaf; $M x C S 1$ expression was also expressed in root and the phloem of stem, but was nearly undetectable in the xylem (Fig. 3A). The results showed that MxCS1 mRNA increased in roots and leaves under a low iron concentration ( $4 \mu \mathrm{M})$ (Fig. $3 B$ ) at the beginning, but decreased little at $48 \mathrm{~h}$; on the contrast, the expression decreased in the roots and leaves under a high iron concentration (160 $\mu \mathrm{M}$; Fig. $3 C)$.

In order to investigate the role of $M x C S 1$ in response to Fe stress, we generated transgenic Arabidopsis plants over-expression of MxCS1 under the control of the CaMV $35 \mathrm{~S}$ promoter. Among 10 transformant lines, six of them (OE-1, OE-2, OE-5, OE-7, OE-8 and OE-9) were confirmed by using RT-PCR analysis, the line of WT (wild-type) and line of vector (transformed only pBI121 vector) were acted as contrapositions (Fig. $4 A$ ).

No significant difference in appearance between WT, vector lines and MxCS1-OE Arabidopsis lines were observed. The $\mathrm{T}_{3}$ transgenic lines $\mathrm{OE}-1$ and $\mathrm{OE}-5$, WT and vector seedlings were placed on MS agar plates supplemented with 4, 100 and $400 \mu \mathrm{M}$ Fe. Up to $100 \mu \mathrm{M}$ Fe, all types of Arabidopsis grow well. WT and vector lines have obvious chlorosis appearance, but MxCS1-OE lines have no obvious chlorosis appearance under Fe-deficiency $(4 \mu \mathrm{M})$. MxCS1-OE lines have better appearance than others on high $\mathrm{Fe}$ concentration $(400 \mu \mathrm{M})$. No difference between WT and vector in response to $\mathrm{Fe}$ stress was observed.

The MxCS1-OE lines had higher fresh mass and higher chlorophyll contents than the WT and vector lines



Fig. 2. Comparison and phylogenetic relationship of MxCS1 with reported citrate synthase proteins from other species. Alignment of MxCS1 with other plant citrate synthase proteins. Positions containing identical residues are shaded in navy blue, while conservative residues are shown in green (top). Panel $A$ (in red lines) - partial alignment of MxCS1 (citrate synthase domain) with other plant citrate synthase proteins. Panel $B$ (in blue lines) - PWPN-box of MxCS1. $C$ - Phylogenetic tree of MxCS1 and other plant citrate synthase proteins. The tree was constructed by the Neighbour-Joining method with $M E G A$ program (v. 4.0). Branch numbers are represented as a percentage of bootstrap values in 1000 sampling replicates and scale indicates branch lengths. 
(Table 1), especially when they were exposed to low or high $\mathrm{Fe}$ concentrations. The MxCS1-OE lines also showed higher $\mathrm{CS}$ activities, and $\mathrm{Fe}$ and citric acid contents (Table 1).

These results suggested that MxCS1 might be one of

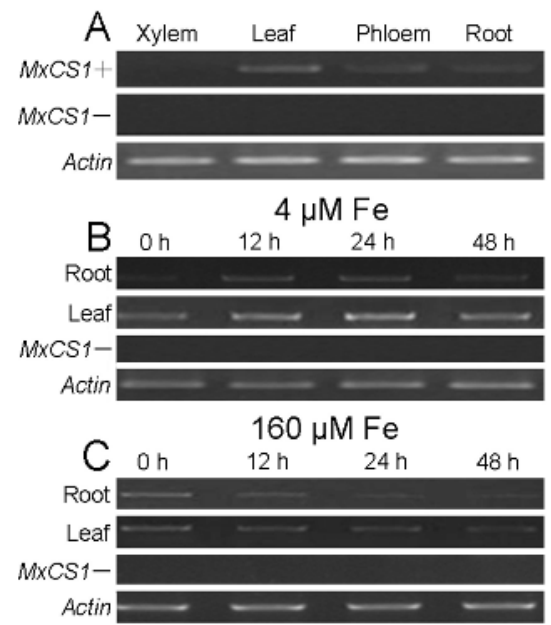

Fig. 3. Expression patterns of MxCS1. A - Expression patterns of $M x C S 1$ in xylem, leaf, phloem and root under normal iron concentration $(40 \mu \mathrm{M}) . B$ - Expression patterns of $M x C S 1$ in a low concentration of $\mathrm{Fe}(4 \mu \mathrm{M})$ in root and leaf at $0,12,2424$ and $48 \mathrm{~h}$. C - Expression patterns of MxCS1 in root and leaf with a high-concentration of $\mathrm{Fe}(160 \mu \mathrm{M})$ at the same time points. Ethidium bromide staining of PCR products using $M x C S 1$-specific primers with (top) and without (middle) prior reverse transcription, and the RT-PCR products of the actin rRNA gene (ApAts1 and ApAtR1 primers) amplified from M. xiaojinensis tissues (bottom). the upstream regulator genes of Fe stress, and the overexpression of MxCS1 can enhance the Fe stress tolerance in Arabidopsis.

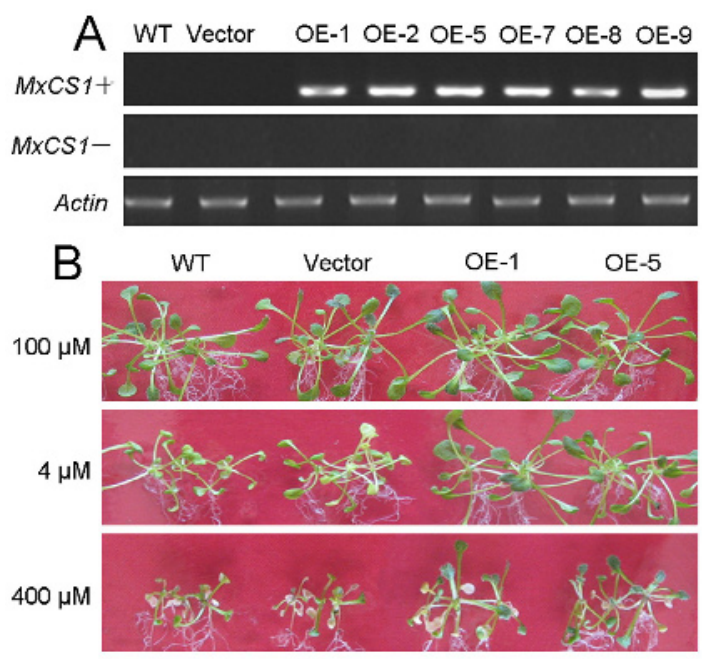

Fig. 4. Expression of MxCS1 in transgenic Arabidopsis (A). The expression level of MxCS1 in wild type (WT), line transformed only PBI121 vector and MxCS1-OE transgenic $\mathrm{T}_{1}$ lines. The results of semi-quantitative RT-PCR. Ethidium bromide staining of PCR products using $M x C S 1$-specific primers with (top) and without (middle) prior reverse transcription, and the PT-PCR products with actin rRNA gene (ApAts1 and ApAtR1) primers (bottom). Over-expression of MxCS1 in Arabidopsis improved Fe tolerance $(B)$. Seedlings of WT, vector and $\mathrm{T}_{3} M x C S 1-\mathrm{OE}$ lines (OE-1 and OE-5) germinated and grown on MS medium supplied with 4, 100 or $400 \mu \mathrm{M}$ Fe for $10 \mathrm{~d}$. All treatments are repeated at least three times and representative results shown here.

Table 1. Effects of different Fe concentrations (4, 100 and $400 \mu \mathrm{M})$ on fresh mass [mg plant ${ }^{-1}$, contents of chlorophyll $\left[\mathrm{mg} \mathrm{g}^{-1}\right.$ (f.m.)], CS activities $\left[\mathrm{U} \mathrm{g} \mathrm{g}^{-1}\right.$ (f.m.)], Fe content $\left[\mathrm{mg} \mathrm{g}^{-1}\right.$ (g.m.)] and citric acid content $\left[\mathrm{mg} \mathrm{g}^{-1}\right.$ (f.m.)] of Arabidopsis transformants and WT. All parameters were measured $10 \mathrm{~d}$ after Fe application. Each value represents the mean of three experiments with 10 replicates in each. Means within a line followed by same letters are not significantly different at $P<0.01$.

\begin{tabular}{|c|c|c|c|c|c|c|c|c|c|c|c|c|}
\hline Parameter & $\begin{array}{l}4 \mu \mathrm{M} \\
\mathrm{WT}\end{array}$ & vector & OE-1 & OE-5 & $\begin{array}{l}100 \mu \mathrm{M} \\
\text { WT }\end{array}$ & vector & OE-1 & OE-5 & $\begin{array}{l}400 \mu \mathrm{M} \\
\text { WT }\end{array}$ & vector & OE-1 & OE-5 \\
\hline Fresh mass & $241 b$ & $253 b$ & $454 a$ & $482 a$ & $521 \mathrm{a}$ & $532 a$ & $545 a$ & $537 \mathrm{a}$ & $127 \mathrm{c}$ & $132 \mathrm{c}$ & $281 \mathrm{~b}$ & $263 b$ \\
\hline Chlorophyll & $0.63 \mathrm{c}$ & $0.61 \mathrm{c}$ & $1.08 \mathrm{~b}$ & $1.11 \mathrm{~b}$ & $0.94 \mathrm{a}$ & $0.97 \mathrm{a}$ & $2.06 \mathrm{a}$ & $2.02 \mathrm{a}$ & $0.97 \mathrm{~b}$ & $1.01 \mathrm{~b}$ & $1.86 \mathrm{a}$ & $1.84 \mathrm{a}$ \\
\hline CS activity & $922 b$ & $961 b$ & $3291 \mathrm{a}$ & $3422 a$ & $523 \mathrm{c}$ & $561 \mathrm{c}$ & $1013 b$ & $1124 b$ & $922 b$ & $934 b$ & $2934 a$ & $2713 a$ \\
\hline Fe content & $42 d$ & $43 d$ & $75 c$ & $79 c$ & $84 \mathrm{c}$ & $85 \mathrm{c}$ & $85 c$ & $86 c$ & $136 \mathrm{~b}$ & $140 \mathrm{~b}$ & $189 \mathrm{a}$ & $187 \mathrm{a}$ \\
\hline Citric acid & $62 \mathrm{c}$ & $66 c$ & $198 \mathrm{a}$ & $201 \mathrm{a}$ & $52 \mathrm{c}$ & $56 \mathrm{c}$ & $101 b$ & $112 b$ & $39 d$ & $41 d$ & $123 b$ & $131 b$ \\
\hline
\end{tabular}

\section{Discussion}

In this study, we isolated a CS protein gene from M. xiaojinensis, designated as MxCS1. The MxCS1 gene encodes a protein of 473 amino acid residues with a predicted molecular mass of $52.5 \mathrm{kDa}$ and theoretical isoelectric point of 8.67. Sequence homologous analysis displayed that there were 86.5 and $92.6 \%$ of nucleotide and amino acid homology between MxCS1 and the CS1 peach gene respectively. The MxCS1 included one conserved $\mathrm{CS}$ domain in the C-terminal region and a PWPN-box in the N-terminus (Takita et al. 1999, Christelle et al. 2002). These results demonstrated that MxCS1 is a novel protein of CS family.

The expression of MxCS1 was enriched more in the leaf than that in root and phloem, but can be hardly detected in the xylem. This expression pattern indicated that $M x C S 1$ may play an important role in active organs. 
When treated with different $\mathrm{Fe}$ concentration, the expression of MxCS1 in roots and leaves were affected markedly. It is possible that MxCS1 plays a key role in regulating Fe stress responses in M.xiaojinensis. Results showed that the content of mRNA of MxCS1 increased in roots and leaves under low Fe treatment after 0, 12 and $24 \mathrm{~h}$, while decreased a little after $48 \mathrm{~h}$. When exposed to Fe-deficiency, $M$. xiaojinensis probably increases the expression of MxCS1 to accelerate the synthesis of CS and citric acid, which consequently promotes the uptake of Fe from poor Fe environment (Tiffin 1970). A possible explanation for the lowered expression of MxCS1 after 48-h treatment is that there has been enough citric acid accumulation for the $\mathrm{Fe}$ absorption at this point. Conversely, the expression of MxCS1 in roots and leaves were down-regulated at high $\mathrm{Fe}$ concentration with the aim to reduce the synthesis of CS and citric acid so that the uptake of Fe from this environment is decreased.

Over-expression of MxCS1 in transgenic Arabidopsis enhanced the tolerance to Fe stress (deficiency as well as surplus). It is possible that $M x C S 1$ plays a crucial role in helping plants to survive from Fe stress by regulating the synthesis of citric acid. Higher content of citric acid in MxCS1-OE Arabidopsis helped to extract Fe from poor Fe environment. Meanwhile, high concentration of citric acid is also helpful in chelating redundant $\mathrm{Fe}$ when plants were exposed to high $\mathrm{Fe}$ (Cataldo et al. 1988, Todorovsky et al. 2002). Therefore, this theory explained why transgenic tobacco showed higher tolerance to high Fe stress. Moreover, the higher Fe content induced by high concentration of citric acid leads to the higher content of chlorophyll in lines over-expressed MxCS1, since $\mathrm{Fe}$ is a necessary for its synthesis.

To our knowledge, this work is the first report on isolation and function characterization of the CS protein gene from Malus. Clarifying the role of the different domains of MxCS1 in stress response will be helpful in breeding stress-resistant Malus by gene transfer. However, further experiments are required to identify other functions of $M x C S 1$ gene.

\section{References}

An, G., Watson, B.D., Chang, C.C.: Transformation of tobacco, tomato, potato, and Arabidopsis thaliana using a binary $\mathrm{Ti}$ vector system. - Plant Physiol. 81: 301-305, 1988.

Aono, M., Kubo, A., Saji, H., Tanaka, K., Kondo, N.: Enhanced tolerance to photo-oxidative stress of transgenic Nicotiana tabacum with high chloroplastic glutathione reductase activity. - Plant Cell Physiol. 34: 129-136, 1993.

Benderliev, K.M., Ivanova, N.I., Pilarskip, P.S.: Singlet oxygen and other reactive oxygen species are involved in regulation of release of iron-binding chelators from Scenedesmus cells. - Biol. Plant. 47: 523-526, 2003.

Bryan, T., Sundar, R.D., Robert, H., Mathieu-Costello, O., Russell, S., Richardson, R.S.: Effect of acute exercise on citrate synthase activity in untrained and trained human skeletal muscle. - Amer. J. Physiol. Regul. Integr. Comp. Physiol. 28: 441-447, 2001.

Cataldo, D.A., McFadden, K.M., Garland, T.R., Wildung, R.E.: Organic constituents and complexation of nickel(II), iron(III), cadmium(II) and plutonium(IV) in soybean xylem exudates. - Plant Physiol. 86: 34-39, 1988.

Christelle, E., Annick, M., Elisabeth, D., Philippe, R., René, M., Christophe, R.: Isolation and characterization of six peach cDNAs encoding key proteins in organic acid metabolism and solute accumulation: involvement in regulating peach fruit acidity. - Physiol. Plant. 114: 259-270, 2002.

Chouliaras, V., Therios, I., Molassiotis, A., Diamantidis, G.: Iron chlorosis in grafted sweet orange (Citrus sinensis L.) plants: physiological and biochemical responses. - Biol. Plant. 48: 141-144, 2004.

Durrett, T.P., Gassmann, W., Rogers, E.E.: The FRD3-mediated efflux of citrate into the root vasculature is necessary for efficient iron translocation. - Plant Physiol. 144: 197-205, 2007.

Green, L.S., Rogers, E.E: FRD3 controls iron localization in Arabidopsis thaliana. - Plant Physiol. 136: 2523-2531, 2004.

Guerinot, M.L., Yi, Y.: Iron: nutritious, noxious and not readily available. - Plant Physiol. 104: 815-820, 1994.

Han, Z.H., Shen, T., Korcak, R.F., Baligar, V.C.: Screening for

iron-efficient species in genus Malus. - J. Plant Nutr. 17: 579-592, 1994a.

Han, Z.H., Wang, Q., Shen, T.: Comparison of some physiological and biochemical characteristics between ironefficient and iron-inefficient species in the genus Malus. - J. Plant Nutr. 17: 1257-1264, 1994b.

Hell, R., Stephan, U.W.: Iron uptake, trafficking and homeoststasis in plants. - Planta 216: 541-551, 2003.

Kojima, I., Iida, C.: Vapor phase digestion of botanical samples with acids in sealed polytetrafluoroethylene bomb. - Anal. Sci. 2: 567-570, 1986.

Li, P., Qi, J.L., Wang, L., Huang, Q.N., Han, Z.H., Yin, L.P.: Functional expression of MxIRT1, from Malus xiaojinensis complements an iron uptake-deficient yeast mutant for plasma membrane targeting via a membrane vesicles trafficking process. - Plant Sci. 171: 52-59, 2006.

Rogers, E.E., Guerinot, M.L.: FRD3, a member of the multidrug and toxin efflux family, controls iron deficiency responses in Arabidopsis. - Plant Cell 14: 1787-1799, 2002.

Stephan, U.W., Schmidke, I., Pich, A.: Phloem translocation of $\mathrm{Fe}, \mathrm{Cu}, \mathrm{Mn}$ and $\mathrm{Zn}$ in Ricinus seedlings in relation to the concentration of nicotianamine, an endogenous chelater of divalent metal ions, in different seedling parts. - Plant Soil 165: 181-188, 1994.

Stephan, U.W., Scholz, G.: Nicotianamine: mediator of transport of iron and heavy metals in the phloem. - Physiol. Plant. 88: 522-529, 1993.

Takahashi, M., Terada, Y., Nakai, I., Nakanishi, H., Yoshimura, E., Mori, S., Naoko, K., Nishizawa, N.K.: Role of nicotianamine in the intracellular delivery of metals and plant reproductive development. - Plant Cell 15: 1263-1280, 2003.

Takita, E., Koyama, H., Shirano, Y., Shibata, D., Hara, T.: Structure and expression of the mitochondrial citrate synthase gene in carrot cells utilizing Al-phosphate. - Soil Sci. Plant Nutr. 45: 197-205, 1999.

Tiffin, L.O.: Translocation of iron citrate and phosphorus in xylem exudate of soybean. - Plant Physiol. 45: 280-283, 1970. 
Todorovsky, D.S., Dumanova, D.G., Todorovska, R.V., Getsova, M.M.: Preparation and characterization of yttriumiron citric acid complexes. - Croat. chem. Acta 75: 155-164, 2002.

Zhang, Y.G., Cheng, J.H., Han, Z.H., Xu, X.F., Li, T.Z.: Comparison of methods for total RNA isolation from Malus xiaojinensis and cDNA amplified using LD-PCR. - China Biotechnol. Bull. 4: 50-53, 2005.

Zhang, Y.G., Kong, J., Wang, Y., Xu, X.F., Liu, L.L., Li, T.Z., Han, Z.H., Zhu, Y.J.: Isolation and characterisation of a nicotianamine synthase gene MxNas1 in Malus xiaojinensis. - J. hort. Sci. Biotechnol. 84:47-52, 2009. 